\title{
Extensional rheometry of cellulose ether solutions: flow instability
}

\author{
Saumil Sudhir Vadodaria $[$ [D $\cdot$ Robert John English
}

Received: 21 August 2015/Accepted: 7 December 2015/Published online: 22 December 2015

(C) The Author(s) 2015. This article is published with open access at Springerlink.com

\begin{abstract}
Capillary breakup extensional rheometry of semi-dilute hydroxyethyl cellulose (HEC) solutions was performed under several step-stretch conditions. The resulting parameters, i.e. terminal steady state extensional viscosity $\left(\eta_{E}\right)$ and the timescale for viscoelastic stress growth, commonly referred to as the extensional relaxation time $\left(\lambda_{E}\right)$ were found to be sensitive to the step-stretch conditions. The $\lambda_{E}$ decreased with increasing step-strain as opposed to the $\eta_{E}$. Prior to the filament break-up, a 'bead-onstring' instability was observed close to the mid-plane. It is believed that this instability originated from the accumulation of viscoelastic stresses near the filament neck leading to the 'elastic recoil' of the extended polymer chains. The reasons for this belief are discussed in detail with the perspective of the past literature. Such type of flow instability has been reported for the first time for a cellulosic system. Various dimensionless numbers were plotted for the HEC solutions and compared with those obtained from past studies for various biopolymers as well as synthetic polymers.
\end{abstract}

Keywords Extensional rheology - CaBER . Cellulose ether · Hydroxyethyl cellulose $\cdot$ Bead-onstring . Flow instability

S. S. Vadodaria $(\bowtie) \cdot$ R. J. English

School of Engineering and Built Environment, Edinburgh

Napier University, Edinburgh, UK

e-mail: saumilvadodaria@gmail.com

\section{Introduction}

Extensional rheometry of polymeric, nanoparticulate and associating fluids has been a subject of interest for many researchers for last three decades following the realization that the deformation regimes in many industrial and biological processes have a significant extensional component (e.g. coating, atomization, flow through porous media and sensory perception in the case of food products) (Duxenneuner et al. 2008; Patruyo et al. 2002). Extensional deformation is encountered whenever a fluid undergoes a change in its cross-sectional area of flow. This includes but is not limited to flow through converging channels, capillary entrance flows and free surface capillary thinning (Gonzalez et al. 2005). Aqueous solutions of biopolymers and their derivatives, owing to their commercial use have to undergo abovementioned extensional deformations and therefore are subjects of interest with regards to their extensional rheological properties.

For fluids with shear viscosity $<10^{3} \mathrm{~Pa}$ s, Capillary break-up extensional rheometry (CaBER) has received much attention during the past few years (Tripathi et al. 2000; Tirtaatmadja et al. 2006; McKinley and Tripathi 2000; Kheirandish et al. 2008; Tripathi et al. 2006; Plog et al. 2005; Arnolds et al. 2010). In CaBER, a fluid filament is subjected to step-strain and the mid-plane diameter of the filament undergoing capillary driven thinning is recorded as a 
function of time (Fig. 1). The user has little control over the elongational flow kinematics and the extensional strain rate is self selected by the balance between capillary, elastic, inertial and viscous forces. For elastic fluids, the diameter decays exponentially whereas for Newtonian fluids (and the viscoelastic fluids in which the polymer chains have been stretched as much as possible during the elastocapillary thinning) the filament diameter decays linearly. The rate constants associated with the exponential and linear capillary thinning allow the determination of an extensional relaxation time $\lambda_{E}$ (timescale for viscoelastic stress growth) and quasi-steady state terminal extensional viscosity $\eta_{E}$ respectively. CaBER measurements are relatively time-efficient and require less sample volume $(<1 \mathrm{ml})$ compared to the techniques such as capillary extrusion (Cogswell 2003), opposed jet flow (Patruyo et al. 2002) and filament stretching rheometry (FiSER) (Bhardwaj et al. 2007). It is noteworthy that Cross-slot rheometry also offers some of the abovementioned advantages of CaBER, and has been used for cellulosic fluids (Sharma et al. 2015) and hyaluronic acid solutions (Haward et al. 2013; Haward 2014). From the perspective of processability, breaking up of an initially stable fluid filament is a process which has commercial applications such as spraying and atomization of pesticides, applying paints and adhesives, coating and food processing operations e.g. container filling (McKinley 2005b).

CaBER technique has been used in the past for Newtonian fluids (Mckinley and Tripathi 2000), shear-thinning viscoelastic fluids (Tripathi et al. 2006; Clasen et al. 2006b; Rodd et al. 2005), nonshear-thinning viscoelastic fluids, i.e. Boger fluids (Plog et al. 2005; Campo-Deano et al. 2011; Anna and McKinley 2001), and yield stress fluids (McKinley 2005a, b; Niedzwiedz et al. 2009) CaBER measurements for a number of systems containing biopolymers and their derivatives in aqueous media have been reported in the past literature, most notably for xanthan (Stelter et al. 2002), hydroxypropyl ether guar (Duxenneuner et al. 2008), methyl hydroxyethyl cellulose (HEC) (Plog et al. 2005), alginate (Storz et al. 2010; Rodriguez-Rivero et al. 2014), scleroglucan (Japper-Jaafar et al. 2009), cellulose acetate + PEO solutions (Chen et al. 2008), cellulose in an ionic liquid 1-ethyl-3-methylimidazolium acetate (Haward et al. 2012) (Michud et al. 2015), DNA (Chevallier
2009), cornstarch-water suspension (Bischoff White et al. 2010), galactomannan (Bourbon et al. 2010), casein/waxy maize starch (Chan et al. 2009) and dilute ethyl HEC solutions (Sharma et al. 2015). In the present study, extensional rheology of HEC solutions has been investigated with the help of CaBER.

The CaBER instrument records the mid-plane filament diameter using a laser sheet micrometer, presupposing that the filament necking takes place in midplane. However, if the filament neck is located somewhere other than the mid-plane, the measurements recorded by the laser sheet micrometer are not reliable. Also, flow instabilities such as 'bead-on-string' are often encountered during capillary thinning experiments. These instabilities refer to the growth of satellite fluid droplet(s) connected to either of or both the fluid hemispheres through the filament. The formation of so called 'bead-on-string' structures in the capillary thinning of filaments and jets has been investigated extensively (Chang et al. 1999; Stelter et al. 2000; Li and Fontelos 2003; Clasen et al. 2006a). 'Bead-onstring' flow instabilities in uniaxial extensional flows have been reported to be primarily caused by either (1) inertia or (2) elasticity.

Rodd et al. (2005) observed that the capillary thinning of low viscosity $\left(\eta_{0}<0.070 \mathrm{~Pa}\right.$ s) fluids with low elasticity is predominantly inertio-capillary in nature. As the pinch-off process is much faster owing to the capillary forces being much stronger compared to the viscous forces, very high elongational deformation rates are attained. At such rates, the inertial stresses dominate over visco-capillary and elastocapillary stresses, resulting into the so-called 'bead(s)on-string' formation. This effect immediately follows the cessation of step-stretch applied by CaBER and is usually accompanied by the oscillations of the hemispherical fluid reservoirs at the endplates. It is very hard to observe a distinctive elastocapillary thinning (exponential decay of the filament) in such filaments and the determination of $\lambda_{E}$ is often not possible (Rodd et al. 2005).

An alternative explanation of the 'bead(s)-onstring' effect can be given by elastic instabilities (Oliveira and McKinley 2005). Chang et al. (1999) predicted using the slender filament equations derived for a FENE (finitely extensible nonlinear elastic) dumbbell model that the accumulated viscoelastic stresses near the mid-filament neck of a thinning filament render it unstable to mechanical wave-like 
(a)

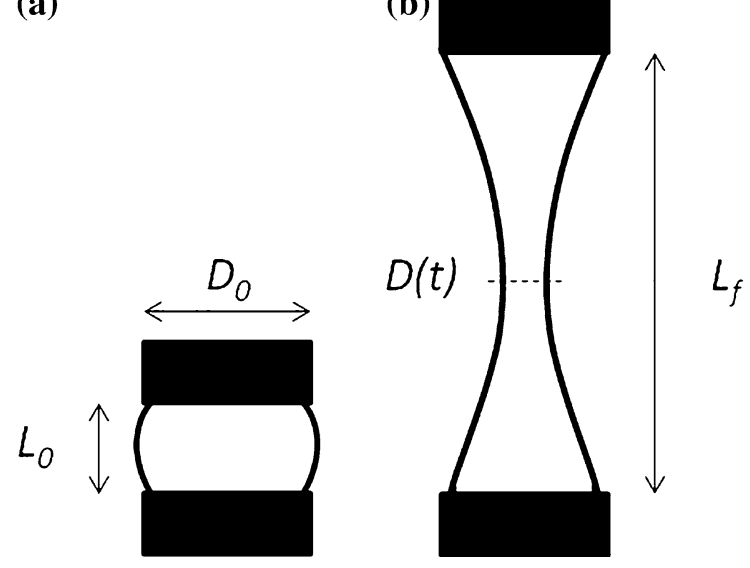

Fig. 1 The endplates of CaBER with sample a before and b after the step-stretch

perturbations. This often triggers flow instability and "elastic recoil" of the polymer chain at the neck near the break-up event. Such local relaxation of elastic stresses leads to the formation of a bead which is connected to the hemispherical fluid reservoirs located at the endplates by (a) thinner and cylindrical filament(s). The polymer molecules are relaxed inside the bead where surface tension dominates, whereas in the filament the polymer molecules are highly stretched and are dominated by viscoelastic stress. Elastocapillary thinning continues in the secondary filament giving rise to another bead, which is connected to one of the hemispherical reservoirs by tertiary filament. Such hierarchical process (also referred to as iterated stretching) repeats itself within the limits of polymer extensibility, which is infinite for Oldroyd-B fluids (Oliveira and McKinley 2005). For real polymeric fluids, elastocapillary thinning is truncated by the finite extensibility of polymer chains, leaving the possibility of the formation of finite number of beads. Also, several criteria have been suggested for the possibility of such flow instability, including extensibility parameters for polymer chains and dimensionless numbers. This phenomenon has been reported experimentally for a flexible polymer such as PEO (Arnolds et al. 2010; Oliveira and McKinley 2005; Oliveira et al. 2006; Sattler et al. 2008), emulsions (Erni et al. 2009), human saliva (Sattler et al. 2012), and for the systems with rigid polymers such as xanthan gum solution drop falling under gravity (Smolka and Belmonte 2006) and DNA suspensions (Juarez and Arratia 2011).
However, since the filament in the vicinity of the midplane is no longer axisymmetric, the observed data following the bead formation cannot be considered reliable for evaluating $\lambda_{E}$ and/or $\eta_{E}$. Also, since cellulosic polymers are widely used as rheology modifiers, an understanding of this flow instability for the systems containing cellulosic polymers is very important due to their potential applications in the commercial processes such as roll-coating, fertilizer spraying, paint-leveling, bottle-filling, misting and inkjet printing (Clasen et al. 2006a; Oliveira and McKinley 2005). These processes have a significant component of free surface extensional deformation and the presence of droplets could potentially affect the quality of the end product. However, according to the best of our knowledge, elastic instabilities such as iterated stretching have never been reported in the past literature for any system containing cellulosic polymers.

In order to identify such flow instabilities, highspeed imaging of the filament decay is essential. Many of the studies concerning biopolymers mentioned above did not provide the high-speed photographic images of their CaBER experiments, which made it very difficult to verify if the filament diameter is smallest at the mid-plane (thereby rendering the use of mid-filament diameter for the analysis questionable) and to identify inertial/elastic flow instabilities, e.g. 'bead-on-string'. The images provided by Duxenneuner et al. (2008) for the capillary thinning of hydroxypropyl ether guar solutions clearly indicate the presence of a 'bead-on-string' instability prior to the break-up, but this effect was not mentioned by the authors in their description of the images. In the present study, an elastic 'bead-on-string' instability observed in the CaBER experiment for HEC solutions has been reported using high-speed photographic image analysis and discussed in detail.

During CaBER experiments, step-stretch conditions can significantly affect the subsequent capillary thinning in a number of respects. A larger step-strain results in a smaller initial filament diameter and early break-up of the filament (Miller et al. 2009). Also, higher stretch speed has shown to stabilize the filament (i.e. increase the maximum length at which the filament exists following the step-stretch) of the fluids composed of casein + waxy maize starch (Chan et al. 2009). These factors eventually affect the rate constants associated with elastocapillary (the exponent) 
and viscocapillary thinning (the slope of the line) of the filament, and hence the determination of $\lambda_{E}$ and $\eta_{E}$. Hence, a comprehensive study of complex fluids with CaBER should include the dependency of $\lambda_{E}$ and $\eta_{E}$ on different step-stretch conditions.

However, most studies concerning CaBER measurements of biopolymers mentioned above have used only one step-stretch profile for the experiments. For synthetic polymers, Miller et al. (2009) have reported that the capillary thinning of $1.0 \mathrm{~g} \mathrm{dl}^{-1}$ aqueous solution of PAA is not significantly sensitive to the step-stretch parameters, while the polymer blends $(\mathrm{PIB}+\mathrm{PDMS})$ and solutions of wormlike micelles exhibit sensitivity (Miller et al. 2009; Kim et al. 2010). In the present study, various step-stretch profiles have been used for the rheometry of an HEC solution and the corresponding parameters related to extensional rheology obtained from the data analysis have been found to be sensitive towards step-stretch parameters.

Capillary thinning of a filament of viscoelastic fluid is governed by the dominant balance of capillarity, inertia, as well as elastic and viscous forces. The relative magnitudes of these forces can be expressed by the respective characteristic timescales (discussed in detail in the "Theory" section). Different dimensionless groups (Deborah, Ohnesorge and Elastocapillary numbers) can be derived using these characteristic timescales. The dimensionless groups are often used to describe the overall nature of the process. However, most literature regarding capillary break-up extensional rheometry of biopolymer related systems lack a detailed force balance analysis of the results.

This study aims to investigate the capillary thinning of semi-dilute HEC solutions with high-speed imaging. The dependency of temporal evolution of filament diameter (and hence the derived parameters $\lambda_{E}$ and $\eta_{E}$ ) on the step-stretch parameters i.e. step strain and stretch speed has been studied. The 'bead-on-string' elastic flow instability observed during the filament thinning has been discussed in detail.

\section{Theory}

Capillary break-up extensional rheometry

CaBER is a rheometrical technique in which, as opposed to most other rheometrical techniques, neither stress nor deformation rate are applied but rather the deformation rates at different stages of the experiment are self-selected by means of force balance between capillary force and other forces such as inertia, elasticity and viscosity. Nevertheless, rheologically important parameters such as stress, strain and strain rate can indeed be calculated by observing the temporal evolution of filament diameter with time and the value of surface tension by means of the following equations suggested by the past literature pioneering experimental development of the technique (McKinley and Tripathi 2000; Anna and McKinley 2001; Stelter et al. 2002; Kolte and Szabo 1999).

$\varepsilon=2 \ln \left(\frac{D_{0}}{D(t)}\right)$

Here $\varepsilon$ is the extensional Hencky strain acquired by the filament, $D_{0}$ is the plate diameter and $D(t)$ is the filament diameter at a given time $t$. The time derivative of Hencky strain gives the effective extensional strain rate:

$\dot{\varepsilon}=2 \frac{d \ln \left(\frac{D_{0}}{D(t)}\right)}{d t}=\frac{-2}{D(t)}\left(\frac{d D(t)}{d t}\right)$

The effective capillary stress at mid-filament is given as:

$\tau=\frac{2 \sigma}{D(t)}$

Here $\sigma$ is the surface tension of the fluid sample which is measured separately. Hence the apparent extensional viscosity is described as the ratio of stress and strain rate, i.e. Eqs. 3 and 2.

$\eta_{E, a p p}=\frac{\tau}{\dot{\varepsilon}}=\frac{-\sigma}{\left(\frac{d D(t)}{d t}\right)}$

It can be noticed from the Eqs. 2 and 4 that the rate of diameter decay is an important factor in capillary breakup extensional rheometry (CaBER). Newtonian and viscoelastic fluids have distinctively different filament thinning profiles. For Newtonian fluids, the elastic stresses are insignificant and hence the extensional flow is primarily caused by a viscocapillary balance (McKinley and Tripathi 2000; Entov and Hinch 1997). The filament radius decreases linearly with a slope of $-\sigma / \eta_{S}$ where $\eta_{S}$ is the shear viscosity 
of the Newtonian fluid. This filament decay can be described by the following expression:

$D(t)=0.1418\left(\frac{\sigma}{\eta_{S}}\right)\left(t_{b}-t\right)$

Here $t_{b}$ is the filament breakup time. The term $\sigma / \eta_{S}$ has a dimension of $\mathrm{m} \mathrm{s}^{-1}$ and is termed as capillary velocity $(V)$. Capillary velocity $V$ is used to calculate capillary number $\mathrm{Ca}$ to describe the importance of capillarity in free-surface flows of Newtonian fluids. However, by its definition, $\mathrm{Ca}$ remains unity during the viscocapillary thinning in CaBER because $V$ is self-selected by the fluid as the ratio $\sigma / \eta_{S}$. The constant prefactor has been introduced by Papageorgiou (1995).

$C a=\frac{\eta_{s} V}{\sigma}=1$

From Eq. 7, the effective strain rate during viscocapillary thinning appears to increase with time due to decreasing filament diameter.

$\dot{\varepsilon}=\frac{2}{D(t)}\left(\frac{\sigma}{\eta_{S}}\right)$

However, in case of Oldroyd-B viscoelastic liquids (Kolte et al. 1997) with a single relaxation time, the filament follows an exponential decay profile in which the dynamics of the filament drainage are controlled by elastocapillary balance, as described in Eq. 8. Here $D_{1}$ is the mid-filament diameter immediately following the step-stretch.

$D(t)=\left(\frac{G D_{1}^{4}}{\sigma}\right)^{1 / 3} \exp \left(\frac{-t}{3 \lambda_{E}}\right)$

Oldroyd-B fluids are models which treat dilute polymer solutions as the suspensions of dumbbells. These dumbbells do not interact with one another and are made of two point masses (beads) connected by a Hookean spring. Upon deformation of the fluid, these dumbbells experience the solvent drag without slip and the spring gets stretched. These infinitely stretchable springs impart elastic stress to the fluid. Provided all the dumbbells have springs with identical force constants, the fluid exhibits a single relaxation time (Clasen et al. 2006a).

Analysis of the data collected enables the determination of $\lambda_{E}$, the characteristic time governing the elasto-capillary thinning (Entov and Hinch 1997;
Liang and Mackley 1994) and $G$, the elastic modulus. Although the constant $\lambda_{E}$ with the dimension of time has been often mentioned in the literature as a 'relaxation time' it is different than the time constants associated with stress relaxation following cessation of steady shear. $\lambda_{E}$ is in fact related to the viscoelastic stress growth as the filament radius approaches zero. This constant is of a wide commercial interest in industrial operations with capillary break-up, such as spraying, mould-filling, jetting etc. (Rodd et al. 2005).

Using Eqs. 2 and 8, the effective extensional strain rate during exponential filament decay can be expressed as an explicit function of the relaxation time $\lambda_{E}$. This implies that the extensional strain rate in the region of elastocapillary thinning is constant and self-selected by the fluid.

$\dot{\varepsilon}=\frac{2}{3 \lambda_{E}}$

Weissenberg number, as defined by the product of strain rate and relaxation time exceeds the critical value of $W i=1 / 2$ for the coil stretch transition in uniaxial flow which ensures that the elastic stress in the filament is balanced by the capillary pressure (Entov and Hinch 1997).

$W i=\dot{\varepsilon} \lambda_{E}=\frac{2}{3}$

Even though the exponential thinning continues indefinitely in the numerical simulations, in practice the finite extensibility of polymer chains limits the elastocapillary stress growth (Stelter et al. 2002). The polymer coils, undergoing strong deformation, get stretched during the extensional flow and induce localized anisotropy in the microstructure at the midfilament. The filament thinning followed by the elongation of chains is dominated by viscocapillary balance in a quasi-steady state. The polymer chains, however, do not necessarily have to undergo full extension up to their contour length. It has been shown that the quasi-steady state is already achieved when the polymer chains are extended to about 1/10th of their full contour length (Yarin 1993).

Force balance, characteristic timescales and dimensionless numbers

Despite of a comprehensive data analysis to obtain the parameters discussed above, a true systematic 
understanding of extensional flows of complex fluids can be very difficult due to various types of forces controlling the overall process. These forces include capillarity, viscous forces, inertia and gravity as well as the elasticity arising from the microstructural deformation within complex fluids. Out of the forces listed above, the relative magnitudes of relevant parameters control the overall nature of the process, and hence it is impossible to describe the process based on any one of them.

A good approach to this problem is to define dimensionless groups which compare the magnitudes of different parameters involved in the process and hence allow us to classify and compare the nature of flows. In a typical CaBER experiment, the filament thinning driven by capillarity is resisted by viscous, inertial and elastic forces. The dominant balance of forces can be determined by comparing different timescales associated with them (Rodd et al. 2005).

\section{Viscous break-up timescale $t_{v}$}

For Newtonian fluids with no inertia or elasticity, the filament decay and subsequent breakup is entirely governed by the viscocapillary force balance. In Eq. 5 , the break-up time $t_{b}$ is identical to $t_{V}$. At $D(t)=0$, i.e. filament breakup, the break-up timescale can be defined as:

$t_{V}=t_{b}=0.1418 \frac{\eta_{0} R_{0}}{\sigma}$

Here $\eta_{0}$ is the so-called zero-shear rate viscosity of the fluid and $R_{0}$ is the endplate radius containing the cylindrical sample which acts as a characteristic length scale in free-surface flows such as capillary thinning in CaBER.

\section{Rayleigh and inertial timescale $t_{R}$ and $t_{i}$}

For an inviscid and inelastic fluid, the filament decay is completely dependent on the inertio-capillary balance. The characteristic time arising from such balance is defined as the Rayleigh timescale (McKinley 2005b).
$t_{R}=\sqrt{\frac{\rho R_{0}^{3}}{\sigma}}$

Here $\rho$ is the fluid density. The Rayleigh timescale is related to the break-up time of an inviscid and inelastic jet $\left(t_{i}\right)$ by the following relationship (Rodd et al. 2005):

$t_{i}=1.95 t_{R}$

\section{Extensional relaxation time $\lambda_{E}$}

When the elastic stresses due to the elongation of the polymer chains in a filament undergoing decay dominate the viscous stresses arising due to the solvent, the exponential thinning as per the Eq. 8 corresponding to the relaxation time $\lambda_{E}$ takes place. Unlike the other timescales discussed above, $\lambda_{E}$ does not ideally relate to the filament break-up time and is ideally independent of the geometry used. Also, it is not a true relaxation time as in context of stress relaxation but a timescale for viscoelastic stress growth.

\section{Deborah number De}

In order to compare the relative magnitudes of both $\lambda_{E}$ and $t_{R}$, their ratio is defined as the Deborah number $(D e)$.

$D e=\frac{\lambda_{E}}{t_{R}}=\frac{\lambda_{E}}{\sqrt{\frac{\rho R_{0}^{3}}{\sigma}}}$

The Eq. 14 suggests that the non-Newtonian effects become more dominant in the free-surface flows such as CaBER at lower characteristic length scales (McKinley 2005b).

\section{Ohnesorge number $\mathrm{Oh}$}

Ohnesorge number compares the relative magnitude of viscous $\left(t_{v}\right)$ and Rayleigh $\left(t_{R}\right)$ timescales:

$\frac{t_{V}}{t_{R}}=\frac{\left(0.1418 \eta_{0} R_{0} / \sigma\right)}{\left(\sqrt{\rho R_{0}^{3} / \sigma}\right)}=\frac{0.1418 \eta_{0}}{\sqrt{\rho R_{0} \sigma}}=0.1418 \mathrm{Oh}$ 


\section{Elastocapillary number Ec}

The collective importance of elastic and capillary stresses to that of the viscous stresses is measured by Elastocapillary number, which can be defined using either $\lambda_{E}$ and $t_{V}$, or De and $O h$ (McKinley 2005b).

$E c=\frac{D e}{O h}=\frac{\lambda_{E}}{\left(t_{V} / 0.1418\right)}=\frac{\lambda_{E} \sigma}{\eta_{0} R_{0}}$

It is again apparent that the non-Newtonian effects become more relevant with diminishing length-scales.

\section{Materials and methods}

Hydroxyethyl cellulose solutions

HEC was supplied under the brand name of Natrosol HEC 250 MR by Hercules Aqualon (now Ashland) with a quoted molecular mass of $M_{w}=720,000 \mathrm{Da}$ in the literature provided with the product. The MS (molecular substitution) value from the product literature provided by the supplier is 2.5 .

1.0, 1.5 and $2.0 \mathrm{~g} \mathrm{dl}^{-1}(\% \mathrm{w} / \mathrm{w})$ solutions of HEC were prepared by mixing the powder with $0.1 \mathrm{M}$ sodium nitrate and $10^{-3} \mathrm{M}$ sodium azide prepared in deionized water. The mixtures were mixed using a roller mixer for $24 \mathrm{~h}$. All solutions then were stored at $21{ }^{\circ} \mathrm{C}$ (room temperature) and were used within $48 \mathrm{~h}$ of preparation.

Density of all solutions was measured using a $25 \mathrm{ml}$ calibrated specific gravity bottle at $22 \pm 0.1^{\circ} \mathrm{C}$. Owing to the relatively small quantities of HEC dissolved in the solutions, the densities were very similar for all concentrations and were considered to be constant at $997 \pm 0.5 \mathrm{~kg} \mathrm{~m}^{-3}$ for all the solutions of HEC.

\section{Shear rheometry}

The shear viscosity was measured at $0.01-1000 \mathrm{~s}^{-1}$ range of shear rate with 30 data points using AR-2000 (TA instruments) controlled stress rheometer fitted with a $2^{\circ}$ steel cone-and-plate geometry with $60 \mathrm{~mm}$ diameter. The solvent trap was employed to delay drying out of the samples. The sample was subjected to pre-shearing for $180 \mathrm{~s}$ at $3 \mathrm{~s}^{-1}$ shear rate followed by $180 \mathrm{~s}$ of equilibration time. Each shear rate was applied for $45 \mathrm{~s}$ and the average shear stress and normal force during last $15 \mathrm{~s}$ were measured. The reproducible nature of all experiments was verified by repeating them. For some samples, the flow curve experiments were repeated with longer time for each shear rate to confirm the steady state.

\section{Tensiometry}

The surface tension of all the samples was measured using a surface Tensiometer K8 (Kruss, Germany) and a platinum DuNuoy ring with $1 \mathrm{~cm}$ radius. The viscosities of all the samples were low enough not to cause appreciable interference during surface tension measurements. The measured surface tension values have been given in Table 2 .

\section{Capillary break-up extensional rheometry}

The extensional rheometry experiments were performed on capillary break-up extensional rheometer (CaBER 1) supplied by Thermo Haake. The endplates with diameter $D_{0}=4 \mathrm{~mm}$ initially separated by $2 \mathrm{~mm}$ (aspect ratio $=0.5$ ) were used for the measurements. This aspect ratio and plate size were chosen with an attempt of restricting Bond Number $B o=\rho g R^{2} / \sigma$ to less than 1. This value of Bond Number indicates the dominance of surface tension over gravity to facilitate the formation of a stable cylindrical bridge between the endplates (Rodd et al. 2005).

For $1.0,1.5$ and $2.0 \mathrm{~g} \mathrm{dl}^{-1}$ solutions, a drop of test fluid was loaded between the plates using a syringe and a gauge. The absence of air bubbles inside the fluid sample was confirmed. The step-strain was adjusted to 1.49 Hencky strain using the micrometer screw provided. The upper plate was moved up with an exponentially increasing velocity within $0.05 \mathrm{~s}$. The evolution of the filament diameter was recorded as a function of time with the laser micrometer as a part of CaBER assembly. Photron MC-1 high-speed camera was used for capturing the videos and images of the filament thinning at 1000 frames $\mathrm{s}^{-1}$.

In order to examine the sensitivity of CaBER measurements to the step-stretch parameters, experiments were performed on $1.5 \mathrm{~g} \mathrm{dl}^{-1}$ solution employing three different step-strains: $1.25,1.50$ and 1.75 . Within each of the step-strain, the three strike times (the timescale during which the step-stretch takes place) of $0.06,0.08$ and $0.10 \mathrm{~s}$ were applied during which the upper plate moved upwards with a constant 
velocity. The reason for choosing to move the plate with constant velocity instead of exponentially increasing velocity is due to the observation of an overshoot in the actuator owing to the velocity of the upper plate being very high near the end of its final position during an exponential strike. At the final position, the plate and the liquid bridge moving with high velocity stop suddenly. As a result, an inertial oscillation moves across the filament bridge from the upper to the lower plate which causes a temporary disruption in the measurement of filament diameter and might also affect the subsequent thinning.

All experiments (shear and extensional rheometry, surface tension measurement) were performed at $25{ }^{\circ} \mathrm{C}$. The image analysis for diameter measurement was performed using Adobe Photoshop CS software.

\section{Size exclusion chromatography}

The molecular mass $M_{W}$ of the HEC sample was measured to be about $7.01 \times 10^{5} \pm 0.3 \%$ Da through aqueous size exclusion chromatography coupled with multi-angle laser light scattering. The sample was dissolved in $0.1 \mathrm{M} \mathrm{NaNO} \mathrm{Na}_{3}+10^{-3} \mathrm{M} \mathrm{NaN}_{3}$ and filtered through Milipore $0.45 \mu \mathrm{m}$ filter. The flow rate of the mobile phase was $0.5 \mathrm{ml} \mathrm{min}{ }^{-1}$ through Suprema mixed bed column containing nearly monodisperse beads of polyhydroxymethacrylate copolymer. Results were treated using Zimm method. Manually measured value of refractive index increment $(\mathrm{d} n / \mathrm{d} c=0.107)$ using Optilab DSP differential refractometer was used in the calculation.

The Radius of gyration $\left(\left\langle R_{g}^{2}\right\rangle^{1 / 2}\right)$ was calculated using the following Zimm equation by plotting $K C / R_{\theta}$ against $\sin ^{2}(\theta / 2)$.

$\frac{K c}{R_{\theta}}=\left(\frac{1}{M}+2 A_{2} c\right)\left(1+\frac{16 \pi^{2} n_{0}^{2}\left\langle R_{g}^{2}\right\rangle}{3 \lambda_{0}^{2}} \sin ^{2} \frac{\theta}{2}\right)$

In the above expression, $K$ is an optical constant, $c$ is the concentration, $R_{\theta}$ is the excess Rayleigh ratio, $\theta$ is the scattering angle, $n_{0}$ is the solvent refractive index, $\lambda_{0}$ is the wavelength of light and $A_{2}$ is the second virial coefficient indicating the thermodynamic solvent quality. The constant $K$ and the excess Rayleigh ratio $R_{\theta}$ can be defined as:
$K=\frac{2 \pi^{2} n_{0}^{2}}{N_{A} \lambda_{0}^{4}}\left(\frac{d n}{d c}\right)^{2}$

Here, $N_{A}$ is the Avogadro number and $\mathrm{d} n / \mathrm{d} c$ is the refractive index increment for the polymer-solvent system, which is measured independently via differential refractometry.

$R_{\theta}=\frac{\left(I_{\theta}-I_{\theta, \text { solvent }}\right) r^{2}}{I_{0} V}=f \frac{E_{\theta}-E_{\theta, \text { solvent }}}{E_{\text {laser }}}$

$I_{\theta}$ and $I_{\theta, \text { solvent }}$ are the scattered light intensity of the solution and the solvent respectively. $V$ is the solution volume and $r$ is the distance between the detector and the scattering volume. Instrumentally, however, $R_{\theta}$ is determined by $E_{\theta}, E_{\theta, \text { solvent }}$ and $E_{\text {laser }}$, the detector signal voltages of the solution, solvent and laser respectively. The value of $\left\langle R_{g}^{2}\right\rangle^{1 / 2}$ manifests the perturbed dimensions of the polymer coil. The parameter called characteristic ratio $\left(C_{\infty}\right)$ compares $\left\langle R_{g}^{2}\right\rangle^{1 / 2}$ to the unperturbed dimensions, hence quantifying the apparent chain stiffness.

$C_{\infty}=\frac{6\left\langle R_{g}^{2}\right\rangle}{N b^{2}}$

Here $b$ is the length of a repeating unit of a polymer chain and $N$ is the degree of polymerization.

The extensibility of a polymer coil decreases with the increasing stiffness of the backbone. Finite extensibility parameter $\left(L^{2}\right)$ can be defined as below:

$L^{2}=3\left(\frac{j \sin ^{2}(\theta / 2) M}{C_{\infty} M_{u}}\right)^{2(1-v)}$

Here $j$ is the number of bonds present in a repeating unit across the bridge connecting two neighboring repeating units on each side (taken 4 for the cellulose repeating unit). $\theta$ is the bond angle between two consecutive repeating units. $M$ and $M_{u}$ are the molecular masses of the polymer and the repeating unit respectively, and $v$ is the excluded volume exponent. There has been at least one report in the literature that the above equation may overpredict experimentally measured values of $L^{2}$ (Szabo et al. 2012).

Based on the above experiments, some of the properties of the HEC sample are summarized in Table 1. 
Table 1 Parameters describing HEC sample

\begin{tabular}{lll}
\hline Parameter name (unit) & Description & Value \\
\hline$M(\mathrm{kDa})$ & Weight average molecular mass of polymer & 701 \\
$M_{u}(\mathrm{kDa})$ & Molecular mass of repeating unit & 0.28 \\
$\left\langle r^{2}\right\rangle^{1 / 2}(\mathrm{~m})$ & Root mean square end-to-end distance (measured) & $6.89 \times 10^{-8}$ \\
$\left\langle R_{g}^{2}\right\rangle^{1 / 2}(\mathrm{~m})$ & Radius of gyration & $2.81 \times 10^{-8}$ \\
$N$ & Average number of repeating units & 2504 \\
$b(\mathrm{~m})$ & Length of repeating unit & $5.15 \times 10^{-10}$ \\
$b N^{I / 2}(\mathrm{~m})$ & & $($ Haward et al. 2012) \\
$C_{\infty}$ & Root mean square end-to-end distance (unperturbed) & $2.58 \times 10^{-8}$ \\
$\Theta$ & Characteristic ratio & 7.15 \\
$v$ & Angle between two repeating units & 110.7 (Zugenmaier 2008) \\
$L^{2}$ & Excluded volume exponent & 0.6 \\
\hline
\end{tabular}

\section{Results and discussion}

\section{Shear rheology}

Figure 2 shows the steady shear viscosity data of all three HEC solutions fitted with the following equation referred to as Cross model:

$\frac{\eta-\eta_{\infty}}{\eta_{0}-\eta_{\infty}}=\frac{1}{1+\left(\gamma^{o} \tau\right)^{m}}$

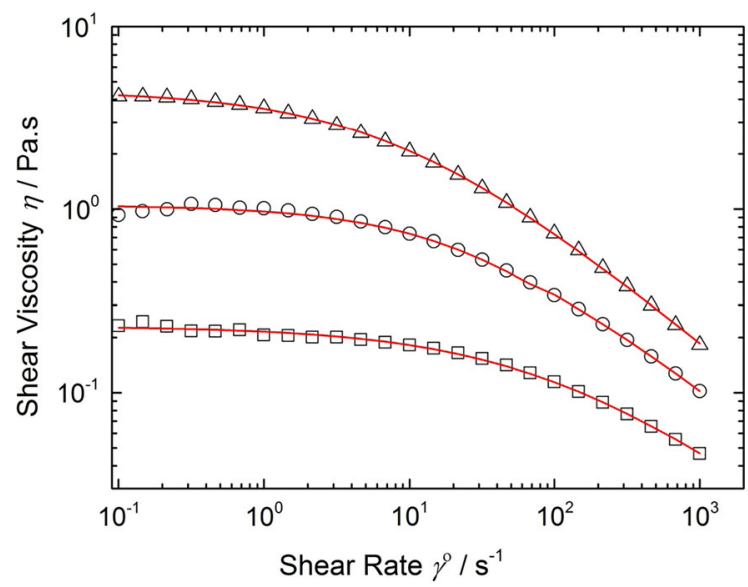

Fig. 2 The shear viscosity of $1.0 \mathrm{~g} \mathrm{dl}^{-1}$ (open square), $1.5 \mathrm{~g} \mathrm{dl}^{-1}$ (open circle) and $2.0 \mathrm{~g} \mathrm{dl}^{-1}$ (open triangle) HEC solutions plotted against shear strain rate. The red lines represent the fits of Cross model for obtaining zero shear viscosity. (Color figure online)
Here $\eta$ is the steady shear viscosity, $\eta_{0}$ and $\eta_{\infty}$ are the so called zero-shear rate viscosity and infinite-shear rate viscosity respectively, $\tau$ is the characteristic time for microstructural change under applied strain conditions and $m$ is the power-law exponent of the shear thinning region (Cross 1965).

\section{CaBER experiments}

The mid-plane diameters of the filaments of 1.0, 1.5 and $2.0 \mathrm{~g} \mathrm{dl}^{-1}$ solutions of HEC undergoing capillary driven thinning are plotted in Fig. 3 against elapsed time where $t=0$ corresponds to the cessation of exponential step-stretching the filament. The measurements obtained from the in-built laser micrometer are in a good agreement with those obtained using image analysis. The filament undergoing thinning remains adequately axiosymmetric according to Fig. 4 which justifies the use of the mid-filament diameter as the representative of the thinning process. A "knee" can be observed in the plots for 1.5 and $2.0 \mathrm{~g} \mathrm{dl}^{-1}$ samples with a duration of $\sim 0.01 \mathrm{~s}$ immediately after the cessation of step-stretch and prior to the beginning of the elastocapillary drainage, indicating very slow decay of the filament within the region immediately following the cessation of step-stretch. The straight red lines represent the fit according to Eq. 8 within the region of exponential decay of the filament. The exponent has been utilized to calculate the extensional relaxation time $\lambda_{E}$, which determines the effective 


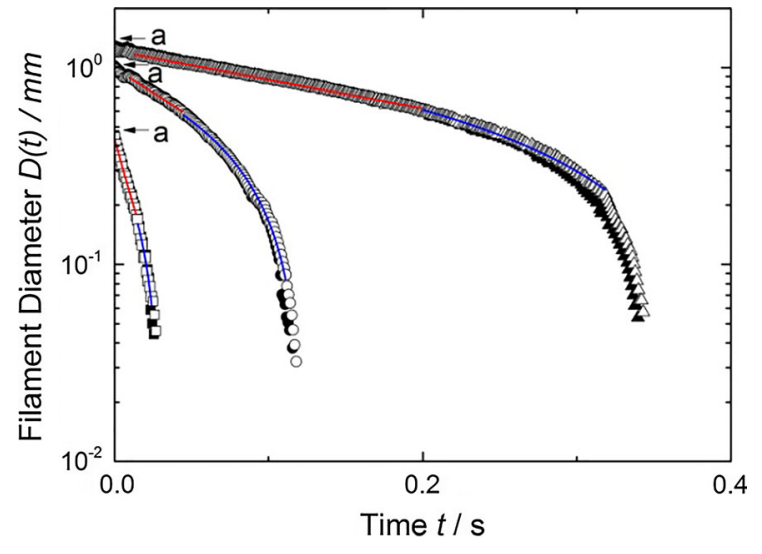

Fig. 3 The diameter of thinning filaments obtained using laser micrometer (open symbols) and image analysis (closed symbols) of $1.0 \mathrm{~g} \mathrm{dl}^{-1}$ (squares), $1.5 \mathrm{~g} \mathrm{dl}^{-1}$ (circles) and $2.0 \mathrm{~g} \mathrm{dl}^{-1}$ (triangles) HEC solutions plotted against time. The red lines and blue lines represent the exponential fit to the data corresponding to the elastocapillary thinning and the linear fit to the data corresponding to the viscocapillary thinning respectively. The initial diameters have been marked with ' $a$ ' for all three concentrations and their images are displayed in Fig. 4. (Color figure online)

self-selected extensional strain rate during the elastocapillary thinning.

The blue lines represent the fit of Eq. 5 describing viscocapillary thinning which follows the elastocapillary thinning once the polymer chains cannot be extended further. The parameters obtained using the fits of Eqs. 8 and 5 have been given in Table 2.

\section{Bead-on-string flow instability}

For all three concentrations, the diameter values close to the break-up event have not been taken into account in the data analysis by Eq. 5 due to a 'bead-on-string' flow instability shown in Fig. 4. The temporal evolution of the filament diameter during viscocapillary thinning for all three concentrations has been plotted against time separately in Fig. 5 with a linear-linear scale. The images corresponding to certain significant events during capillary thinning (i.e. cessation of stepstretch, flow instability and filament break-up) have been shown in Fig. 4. All the HEC samples under investigation were observed to form only one bead prior to the break-up event.

As discussed in the introduction, these flow instabilities can be explained using either inertial or elastic effects. In the past literature, several criteria related to the fluid properties and the capillary thinning have been identified to be associated with the type of 'beadon-string' instability.

Rodd et al. (2005) reported that it is possible to observe a 'bead-on-string' flow instability immediately following the cessation of step-stretch of a fluid filament (low viscosity PEO solutions) with $O h \ll 1$. Also, the apparent absence of the region of exponential filament decay makes it very difficult to calculate $\lambda_{E}$ and shows that the elastic effects are very weak $(D e \ll 1)$. With capillary and inertial forces dominating over viscous and elastic forces, the fluid hemispheres attached to each plate undergo oscillation with a characteristic timescale proportional to $t_{R}$ (Rodd et al. 2005). However, the data corresponding to the filament decay in Fig. 3 for all three concentrations contain easily recognizable regions of exponential thinning, and hence the elastic effects are not insignificant. This is further confirmed by the dimensional analysis performed later in this paper where $0.44 \leq D e \leq 8.78$. The intermediate range of Ohnesorge number $(0.63 \leq O h \leq 14.14)$ indicates that the filament decay is not completely dominated by inertiocapillary forces, as opposed to the data reported by Rodd et al. (2005).

An alternative explanation of such 'bead-on-string' instabilities have been given by Chang et al. (1999) as well as Oliveira and McKinley (2005). The accumulated viscocapillary stress at the filament neck renders the filament unstable to mechanical perturbations near the break-up event. The elongated polymer coils may eventually undergo 'elastic recoil' to generate 'beadon-string' structures. Chang et al. (1999) and Oliveira and McKinley (2005) proposed key conditions to be met by certain physical parameters (i.e. $\mathrm{De}, \mathrm{Oh}$, viscosity ratio $S=\eta_{s} / \eta_{0}$ where $\eta_{S}$ is the solvent viscosity, and the finite extensibility parameter $L^{2}$ indicating the ratio of maximum extended length to the equilibrium length of polymer coils) for the possibility of such elastic instability. They proposed De $\gg 1$ to ensure sufficient elastic effects, $S \neq 0$ or 1 for an intermediate viscosity ratio and $L^{2} \gg 1$ to ensure that the iterated nature of elastic elongation and recoil processes is not truncated by limited molecular extensibility.

In order to calculate these values (given in Table 3) for the systems being investigated in this study, some of the values from Table 2 were used.

As it is apparent from Table 2, the value of $L^{2}$ remains much above 1 , as the criteria defined by 
Fig. 4 Filament thinning of 1.0, 1.5 and $2.0 \mathrm{~g} \mathrm{dl}^{-1} \mathrm{HEC}$ solutions at different times. The red lines indicate midfilament plane where the diameter is measured using in-built laser micrometer. The corresponding data points have been marked in Fig. 4. Six images for each concentration represent several important events during capillary thinning. (Color figure online)

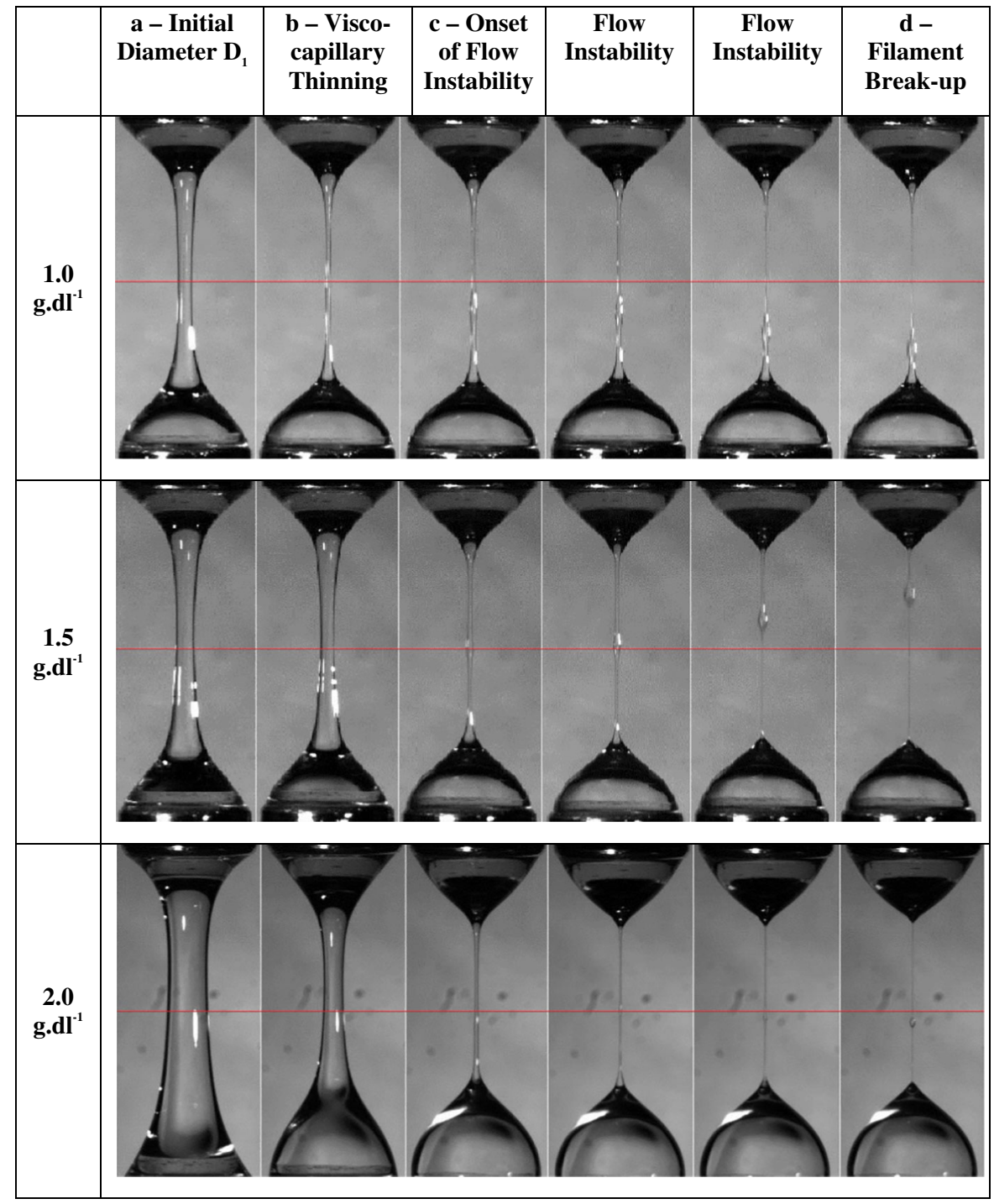

Table 2 Parameters obtained from the surface tension and capillary thinning measurements of the HEC solutions at three different concentrations

\begin{tabular}{llllll}
\hline $\begin{array}{l}\text { HEC concentration } \\
\left(\mathrm{g} \mathrm{dl}^{-1}\right)\end{array}$ & $\begin{array}{l}\text { Surface tension } \\
\left(\mathrm{N} \mathrm{m}^{-1}\right)\end{array}$ & $\begin{array}{l}\eta_{O} \\
(\mathrm{~Pa} \mathrm{~s})\end{array}$ & $\begin{array}{l}\text { Experimental breakup time } \\
(\mathrm{s})\end{array}$ & $\begin{array}{l}\text { Terminal } \eta_{E} \\
(\mathrm{~Pa} \mathrm{~s})\end{array}$ & $\begin{array}{l}\text { Relaxation time } \lambda_{E} \\
(\mathrm{~s})\end{array}$ \\
\hline 1.0 & 0.058 & 0.215 & 0.029 & 1.75 & 0.00532 \\
1.5 & 0.054 & 1.35 & 0.118 & 2.90 & 0.0303 \\
2.0 & 0.050 & 4.47 & 0.346 & 6.36 & 0.098 \\
\hline
\end{tabular}

Chang et al. (1999) and Oliveira and McKinley (2005), as does the value of $S$ unequal to 0 and 1 according to Table 3. However, De for $1.0 \mathrm{~g} \mathrm{dl}^{-1}$ sample remains below 1 , as opposed to those criteria. In a later publication, it was theoretically shown that systems with $D e<1$ are also prone to 'bead-onstring' instability (Bhat et al. 2010).

In Fig. 5, the data points after the onset of flowinstability have been excluded from the calculation of linear fits for all three concentrations as the observed 


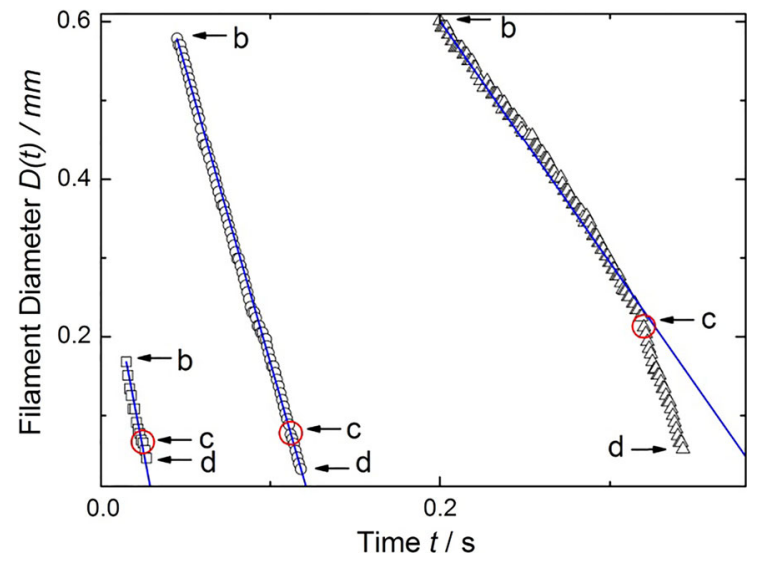

Fig. 5 The diameter versus time data corresponding to the viscocapillary thinning has been plotted on a linear-linear axes graph for 1.0 (squares), 1.5 (circles) and 2.0 (triangles) $\mathrm{g} \mathrm{dl}^{-1}$ HEC solutions. The onset of viscocapillary thinning, the onset of flow instability and the filament break-up event for each concentration have been marked with ' $b$ ', ' $c$ ' and ' $d$ '. The blue lines represent linear fit to the data between the line segment ' $b c$ ' according to Eq. 5. (Color figure online)

flow instability cannot be classified as viscocapillary thinning. However, it is noteworthy that the extended lines for data fit still remain in good agreement with the observed data within the region of flow instability for 1.0 and $1.5 \mathrm{~g} \mathrm{dl}^{-1}$ solutions but not for the $2.0 \mathrm{~g} \mathrm{dl}^{-1}$ solution.

\section{Effect of step-stretch parameters}

In CaBER experiments, the capillary thinning is often influenced by the applied conditions of step-stretch (Miller et al. 2009). After the cessation of step-stretch, the initial diameter $D_{1}$ is influenced by the stretch ratio $L_{f} / L_{0}$ (where $L_{f}$ is the final height and $L_{0}$ is the initial height of the sample) according to the following "reverse squeeze flow" solution (Anna and McKinley 2001; McKinley and Sridhar 2002):

$$
\left(\frac{D_{0}}{D_{1}}\right)^{4 / 3}=\left(\frac{L_{f}}{L_{0}}\right)
$$

According to Eq. 23, $D_{1}$ decreases with increasing step-strain. Moreover, $D_{1}$ decreases further with increasing stretch-time as the fluid across the filament drains under the influence of gravity. The variation in $D_{1}$ influences the elastocapillary thinning and the filament break-up time. Hence the parameters such as $\lambda_{E}$ and $\eta_{E}$ which are derived from the exponential and linear decay of the filament respectively, vary appreciably with the stretch profile. The results of CaBER experiments should therefore be interpreted and compared in the context of the applied step-stretch profile.

The variation in the exponent of elastocapillary filament decay and the slope of viscocapillary filament decay (according to Eqs. 8 and 5 respectively) with the step-stretch parameters can be observed in Table 4 . The $D_{1}$ values for all strike times within a step strain are lower compared to the calculated $D_{1}$ as per Eq. 23, indicating significant axial flow under gravity. The exponent for elastocapillary thinning generally tends to decrease with decreasing $D_{1}$. Also the slope of linear filament decay generally tends to increase with decreasing filament break-up time.

The temporal evolution of filament diameter of three different CaBER experiments with same stretch time but different step-strain is plotted in Fig. 6a. The data points acquired following the onset of 'bead-onstring' flow instability have been neglected during the analysis. However, according to Fig. 6b, the neglected

Table 3 Dimensionless groups and other parameters calculated for HEC

\begin{tabular}{lll}
\hline Parameter (concentration in $\left.\mathrm{g} \mathrm{dl}^{-1}\right)$ & Description (concentration in $\mathrm{g} \mathrm{dl}^{-1}$ ) & Value \\
\hline De $(1.0)$ & Deborah number (1.0) & 0.440 \\
Oh $(1.0)$ & Ohnesorge number (1.0) & 0.630 \\
$S(1.0)$ & Ratio of solvent viscosity to solution viscosity for 1.0 & $4.61 \times 10^{-3}$ \\
$D e(1.5)$ & Deborah number (1.5) & 2.15 \\
Oh $(1.5)$ & Ohnesorge number (1.5) & 3.45 \\
$S(1.5)$ & Ratio of solvent viscosity to solution viscosity for 1.5 & $8.7 \times 10^{-4}$ \\
$D e(2.0)$ & Deborah number (2.0) & 8.78 \\
$O h(2.0)$ & Ohnesorge number (2.0) & 14.1 \\
$S(2.0)$ & Ratio of solvent viscosity to solution viscosity for 2.0 & $2.21 \times 10^{-4}$ \\
\hline
\end{tabular}


Table 4 The initial filament diameter $D_{1}$ following the cessation of step-stretch, the filament breakup time $t_{b}$, the exponent values calculated from the exponential fit for the data in elastocapillary thinning region and the slope calculated from the linear fit for the data in viscocapillary thinning region for $1.5 \mathrm{~g} \mathrm{dl}^{-1} \mathrm{HEC}$ solutions at various step-stretch conditions

\begin{tabular}{|c|c|c|c|c|c|c|}
\hline $\begin{array}{l}\text { Step Hencky } \\
\text { strain }\end{array}$ & $\begin{array}{l}\text { Strike } \\
\text { time/ms }\end{array}$ & $\begin{array}{l}\text { Measured initial } \\
\text { diameter } D_{1} / \mathrm{mm}\end{array}$ & $\begin{array}{l}\text { Break-up time } \\
t_{b} / \mathrm{s}\end{array}$ & $\begin{array}{l}\text { Exponent } \\
\left(-1 / 3 \lambda_{E}\right)\end{array}$ & $\begin{array}{l}\text { Slope } \\
\left(-0.142 \times \sigma / \eta_{s}\right)\end{array}$ & $\begin{array}{l}\text { Reverse squeeze } \\
\text { flow } D_{1} / \mathrm{mm}\end{array}$ \\
\hline \multirow[t]{3}{*}{1.25} & 60 & 1.360 & 0.1108 & -12.33 & -0.01405 & \multirow[t]{3}{*}{1.56} \\
\hline & 80 & 1.275 & 0.0924 & -19.32 & -0.01586 & \\
\hline & 100 & 1.140 & 0.0965 & -18.46 & -0.01270 & \\
\hline \multirow[t]{3}{*}{1.50} & 60 & 0.870 & 0.0800 & -20.91 & -0.01045 & \multirow[t]{3}{*}{1.29} \\
\hline & 80 & 0.600 & 0.0477 & -38.49 & -0.01133 & \\
\hline & 100 & 0.475 & 0.0380 & -52.24 & -0.01251 & \\
\hline \multirow[t]{3}{*}{1.75} & 60 & 0.590 & 0.0580 & -31.31 & -0.00898 & \multirow[t]{3}{*}{0.89} \\
\hline & 80 & 0.510 & 0.0508 & -37.89 & -0.00886 & \\
\hline & 100 & 0.390 & 0.0401 & -46.14 & -0.00829 & \\
\hline
\end{tabular}

data nevertheless seems to be in agreement with the linear fit. It is evident from the data fits (red and blue lines) from both Fig. 6a, b that the rate constants associated with both exponential and linear filament decay vary with step-stretch profile. It is thus apparent that the parameters derived from these rate constants also vary with step-stretch parameters. In Fig. 7, the variation in $\lambda_{E}$ and $\eta_{E}$ has been plotted with relevant error bars describing the reproducibility of the experiments.

The variation in $\eta_{E}$ is relatively small but significant, whereas $\lambda_{E}$ strongly depends upon the step-

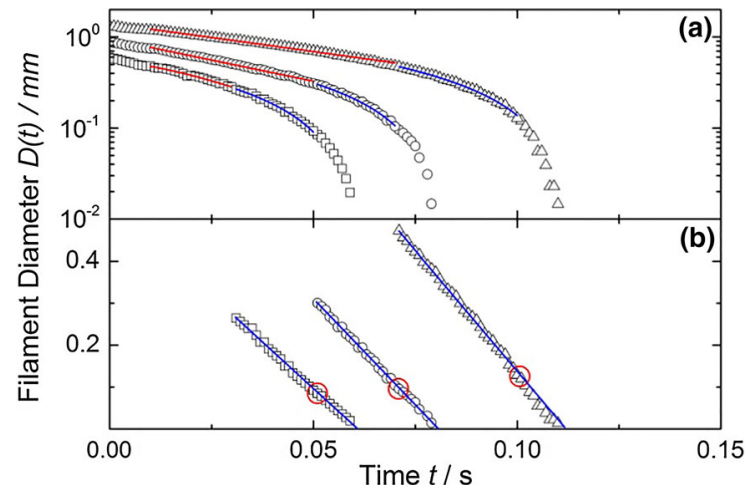

Fig. 6 a Diameter versus time data for $1.5 \mathrm{~g} \mathrm{dl}^{-1} \mathrm{HEC}$ solution using $60 \mathrm{~ms}$ stretch time and different step-Hencky strain values such as 1.25 (triangles), 1.50 (circles) and 1.75 (squares) on a log-linear plot. The red and blue lines indicate the exponential and linear fits to the data corresponding to elastocapillary and viscocapillary thinning. b The segments with viscocapillary thinning and flow instability taken from the curves displayed in a on a linear-linear plot. The graph legend is similar to that of a. The red circles indicate the onset of flow instability and the subsequent diameter values have not been taken into account in fitting the straight line. (Color figure online) stretch conditions according to Fig. 7. Also, $\lambda_{E}$ decreases with increasing step-strain while $\eta_{E}$ decreases. Miller et al. (2009) found that while the step-stretch parameters affected the measurement of extensional viscosity and relaxation time of wormlike micelle solutions and PIB/PDMS polymer blends, similar measurements for $1.0 \mathrm{~g} \mathrm{dl}^{-1}$ aqueous solution of polyacrylamide were insensitive towards the stretch conditions (Miller et al. 2009).

Thus, even though the capillary drainage of the filaments of complex fluids is dominated by the balance of capillary, elastic, inertial and viscous forces in CaBER, it is apparently important to determine the sensitivity of parameters such as $\eta_{E}$ and $\lambda_{E}$ to stepstretch profiles in CaBER measurements. However, according to the best of our knowledge, there have been no reports in the literature about the sensitivity of capillary thinning of polysaccharide solutions to the step-stretch conditions in CaBER measurements.

\section{Dimensionless groups}

Dimensionless groups such as $D e$ for various polymeric systems reported in the past literature and HEC have been plotted in Fig. 8 against their corresponding $O h$. The straight lines on the plot indicate various values for $E c=D e / O h$. For $1.5 \mathrm{~g} \mathrm{dl}^{-1} \mathrm{HEC}$ solution with different step-stretch profiles, the variation in $D e$ is observed while $O h$ remain constant. This is obvious because the calculation of $O h$ involves neither $\eta_{E}$ nor $\lambda_{E}$.

Rodd et al. (2005) suggested that for a polymer solution with either of the conditions, i.e. $\lambda_{E}<0.001 \mathrm{~s}$ 
Fig. 7 Extensional relaxation time $\lambda_{E}$ (red squares with error bars) and steady state extensional viscosity $\eta_{E}$ (blue squares with error bars) measured within the region of viscocapillary thinning is plotted against different strike times for different values of initially applied step strains. The dotted lines guide the eyes. Error bars represent one standard deviation. (Color figure online)

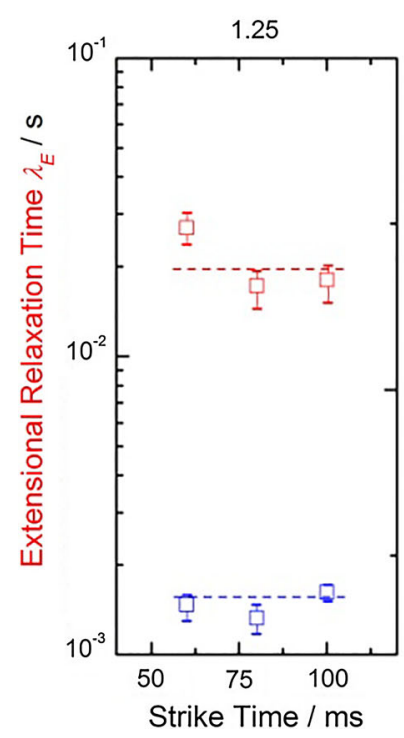

Step Hencky Strain

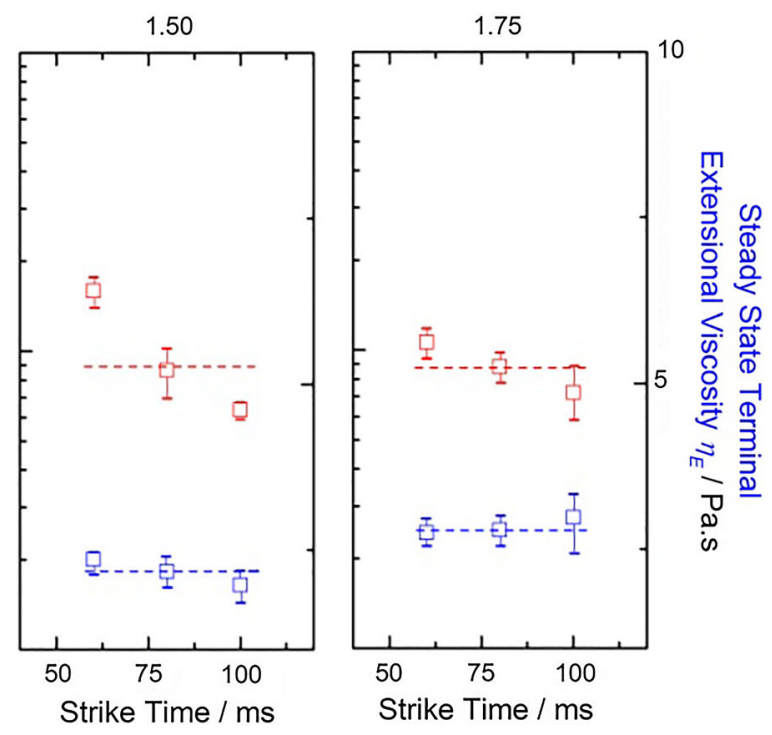

or $\eta_{0}<0.070 \mathrm{~Pa} \mathrm{~s}$, the inertial effects dominate over the elastic/viscous effects and CaBER measurement will not be possible (Rodd et al. 2005). These parameters corresponded to the dimensionless groups $D e<1$ and $O h<0.14$. These restrictions were referred to as the "operability diagram". McKinley et al. (2005) proposed that CaBER tests become impractical for the polymer solutions with $D e<1$ and $O h<1$ (McKinley 2005a). However, many data points in Fig. 8 correspond to $D e<1$ and $O h<1$. Moreover, $\eta_{0}$ of hydroxypropyl ether guar solutions at various concentrations ranged from 0.0012 to $0.596 \mathrm{~Pa} \mathrm{~s}$ which included four concentrations with $\eta_{0}<0.070$ Pa.s. Duxenneuner et al. (2008) reported that all of these solutions exhibited elastocapillary thinning according to Eq. 8 and the calculation of $\lambda_{E}$ was possible.

This can be explained by the fact that the characteristic length scale (i.e. plate radius $R_{0}$ ) used to calculate the characteristic timescales and consecutively "global" values of $D e$ and $O h$ does not consider the continuous filament thinning. Hence with the diminishing length scale (i.e. filament diameter) the inertial effects become increasingly less important and the "local" $\mathrm{De}$ and $\mathrm{Oh}$ increase. Also, since the parameters related to extensional rheology of a nonNewtonian fluid cannot be determined using its shear rheology data, the predictions about the feasibility of CaBER experiments using $\eta_{0}$ should be made carefully.

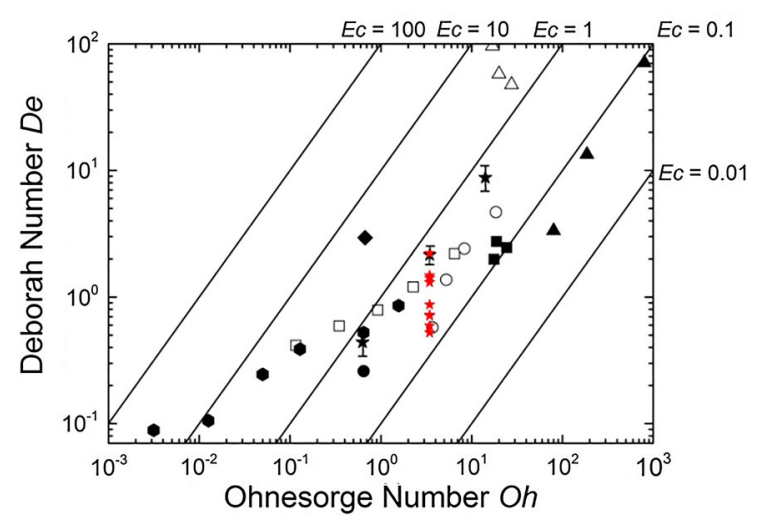

Fig. 8 Deborah number as a function of Ohnesorge number for HEC solutions (black star) measured during this study and various other systems from past literature such as PEO (open square) (Arnolds et al. 2010), alginate (black square) (Storz et al. 2010), hydroxypropyl ether guar (black hexagon) (Duxenneuner et al. 2008), scleroglucan (black circle) (Japper-Jaafar et al. 2009), methyl hydroxyethyl cellulose with different molecular mass distribution (open triangle) (Plog et al. 2005), cellulose in an ionic liquid (black triangle) (Haward et al. 2012), methacrylic acid + acrylic acid + ethyl acrylate copolymer (open circle) (Kheirandish et al. 2008) and DNA (black rhombus) (Chevallier 2009). For $1.5 \mathrm{~g} \mathrm{dl}^{-1}$ HEC solution, the data points (red star) calculated using a range of step-stretch profiles have been plotted. The straight lines indicate different values of elastocapillary numbers ranging from 0.01 to 100 . Error bars represent one standard deviation. (Color figure online)

However, these dimensionless groups are nevertheless useful for determining the dominant balance of forces in the flow. The $E c$ being a ratio of $\mathrm{De}$ to $\mathrm{Oh}$ 
indicates the dominant effect in the filament thinning process. For $E c$ below and above unity, the filament thinning and necking is viscously-dominated and elastically-dominated respectively (Clasen et al. 2006b; McKinley 2005a). While it is evident from Fig. 8 that elastocapillary thinning can occur for a measurable duration even when $E c<1$, the transition from the viscocapillary balance (immediately after the step-strain) to the elastocapillary balance shifts to later times with decreasing $E c$.

In Fig. 8, the $E c$ of HPG and PEO solutions increases with concentration while that of the HEC, methacrylic acid + acrylic acid + ethyl acrylate copolymer and cellulose in the ionic liquid 1-ethyl-3-methylimidazolium acetate solutions remain almost constant with their concentration. Decreasing $E c$ with increasing concentration is apparently counter-intuitive because stronger elastic effects (with higher $\lambda_{E}$ ) are expected with increasing concentration. However, in certain systems the increase in viscosity is greater than the increase in relaxation time. This phenomenon has been previously observed for polystyrene in diethyl phthalate (Clasen 2010) and PEO in water (Sachsenheimer et al. 2014).

\section{Conclusion}

Capillary break-up extensional rheology of HEC solutions was investigated at three different concentrations (1.0, 1.5 and $\left.2.0 \mathrm{~g} \mathrm{dl}^{-1}\right)$ and the parameters such as steady state terminal extensional viscosity and extensional relaxation time were determined. The high-speed imaging of filament thinning process showed flow instability (i.e. 'bead-on-a-string') near the filament break-up event. It was proposed that this flow instability emerged from "elastic recoil" of polymer chains subsequently causing thinning of the "secondary filament" formed between the "bead" from elastic recoil and the hemispheres of the liquid bridge. This phenomenon has been reported for the first time for any cellulosic system. While many other polymeric systems may also exhibit this effect, widespread use of cellulose ethers as rheology modifiers makes such findings commercially relevant.

The sensitivity of CaBER measurements to stepstretch parameters for cellulosic systems was also reported for the first time. $1.5 \mathrm{~g} \mathrm{dl}^{-1} \mathrm{HEC}$ solution was examined by applying three different timescales for step-strain within each of the three step-strains with different magnitude. It was observed that the stepstretch profiles influenced the rate constants associated with exponential and linear decay of the filament as well as the filament break-up time. The variation in these rate constants affected the extensional relaxation time and the steady state extensional viscosity. The relaxation time was observed to decrease and the extensional viscosity was observed to increase with increasing stepstrain. The 'reverse squeeze flow' solution was applied to various step-strains and the theoretical initial diameter values for various step-strains were calculated and compared with the observed values.

Different characteristic timescales (inertial, elastic and viscous) which are important for capillary thinning and breakup were discussed. The force balance as described by the dimensionless numbers (Deborah, Ohnesorge and Elastocapillary) derived from these timescales was explained.

De was plotted against $\mathrm{Oh}$ for a variety of biopolymers and synthetic polymers from the previous literature and the HEC solutions examined in this study. The variation of $E c$ with concentration for different systems was explained using the competing effects of extensional relaxation time and viscosity increasing with the concentration.

Open Access This article is distributed under the terms of the Creative Commons Attribution 4.0 International License (http:// creativecommons.org/licenses/by/4.0/), which permits unrestricted use, distribution, and reproduction in any medium, provided you give appropriate credit to the original author(s) and the source, provide a link to the Creative Commons license, and indicate if changes were made.

\section{References}

Anna S, McKinley G (2001) Elasto-capillary thinning and breakup of model elastic liquids. J Rheol 45:115-138

Arnolds O, Buggisch H, Sascsenheimer D, Willenbacher N (2010) Capillary breakup extensional rheometry (CaBER) on semi-dilute and concentrated polyethylene oxide (PEO) solutions. Rheol Acta 49(11-12):1207-1217

Bhardwaj A, Richter D, Chellamuthu M, Rothstein J (2007) The effect of pre-shear on the extensional rheology of wormlike micelle solutions. Rheol Acta 46:861-875

Bhat P, Appathurai S, Harris M, Pasquali M, McKinley G, Basaran O (2010) Formation of beads-on-a-string structures during breakup of viscoelastic filaments. Nat Phys 6:625-631

Bischoff White E, Chellamuthu M, Rothstein J (2010) Extensional rheology of a shear-thickening cornstarch and water suspension. Rheol Acta 49(2):119-129 
Bourbon A, Pinheiro A, Ribeiro C, Miranda C, Maia J, Teixeira J et al (2010) Characterization of galactomannans extracted from seeds of Gleditsia triacanthos and Sophora japonica through shear and extensional rheology: comparison with guar gum and locust bean gum. Food Hydrocoll 24(2-3):184-192

Campo-Deano L, Galindo-Rosales F, Oliveira M, Alves M, Pinho F (2011) Boger fluid flow through hyperbolic contraction microchannels. In: 3rd micro and nano flows conference, Thassaloniki, Greece

Chan P, Chen J, Ettelaie R, Alevisopoulos S, Day E, Smith S (2009) Filament stretchability of biiopolymer fluids and controlling factors. Food Hydrocoll 23:1602-1609

Chang H, Demekhin E, Kalaidin E (1999) Iterated stretching of viscoelastic jets. Phys Fluids 11(7):1717-1737

Chen L, Bromberg L, Hatton A, Rutledge G (2008) Electrospun cellulose acetate fibres containing chlorhexidine as a bactericide. Polymer 49(5): 1266-1275

Chevallier C (2009) Écoulements élongationnels de solutions diluées de polymères, Ph.D. thesis

Clasen C (2010) Capillary breakup extensional rheometry of semi-dilute polymer solutions. Korea-Australia Rheol J 22(4):331-338

Clasen C, Eggers J, Fontelos M, Li J, McKinley G (2006a) The beads-on-string structure of viscoelastic threads. J Fluid Mech 556:283-308

Clasen C, Plog J, Kulicke W, Owens M, Macosko C, Scriven L et al (2006b) How dilute are dilute solutions in extensional flows? J Rheol 50(6):849-881

Cogswell F (2003) Polymer melt rheology: a guide for industrial practice. Woodhead Publishing Limited, Cambridge

Cross M (1965) Rheology of non-Newtonian fluids: a new flow equation for pseudoplastic systems. J Colloid Sci 20:417-437

Duxenneuner MR, Fischer P, Windhab EJ, Cooper-White JJ (2008) Extensional properties of hydroxypropyl ether guar gum solutions. Biomacromolecules 9:2989-2996

Entov V, Hinch E (1997) Effect of a spectrum of relaxation times on the capillary thinning of a filament of elastic liquid. J Non-Newton Fluid Mech 72:31-53

Erni P, Cramer C, Marti I, Windhab E, Fischer P (2009) Continuous flow structuring of anisotropic biopolymer particles. Adv Colloid Interface Sci 150:16-26

Gonzalez J, Muller A, Torres M, Saez A (2005) The role of shear and elongation in the flow of solutions of semi-flexible polymers through porous media. Rheol Acta 44:396-405

Haward S (2014) Characterization of hyaluronic acid and synovial fluid in stagnation point elongational flow. Biopolymers 101:287-305

Haward S, Sharma V, Butts C, McKinley G, Rahatekar S (2012) Shear and extensional rheology of cellulose/ionic liquid solutions. Biomacromolecules 13(5):1688-1699

Haward S, Jaishankar A, Oliveira M, Alves M, McKinley G (2013) Extensional flow of hyaluronic acid solutions in an optimized microfluidic cross-slot device. Biomicrofluidics 7:044108

Japper-Jaafar A, Escudier M, Poole R (2009) Turbulent pipe flow of a drag-reducing rigid 'rod-like' polymer solution. J Non-Newton Fluid Mech 161(1-3):86-93

Juarez G, Arratia P (2011) Extensional rheology of DNA suspensions in microfluidic devices. Soft Matter 7:9444
Kheirandish S, Guybaidullin I, Wohlleben W, Willenbacher N (2008) Shear and elongational flow behavior of acrylic thickener solutions. Rheol Acta 47(9):999-1013

Kim N, Pipe C, Ahn K, Lee S, McKinley G (2010) Capillary breakup extensional rheometry of a wormlike micellar solution. Korea-Aust Rheol J 22(1):31-41

Kolte M, Szabo P (1999) Capillary thinning of polymeric filaments. J Rheol 43(3):609-626

Kolte M, Rasmussen H, Hassager O (1997) Transient filament stretching rheometer II: numerical simulation. Rheol Acta 36(3):285-302

Li J, Fontelos M (2003) Drop dynamics on the beads-on-string structure for viscoelastic jets: a numerical study. Phys Fluids 15(4):922-937

Liang R, Mackley M (1994) Rheological characterization of the time and strain dependence for polyisobutylene solutions. J Non-Newton Fluid Mech 52:387-405

McKinley G (2005a) Dimensionless groups for understanding free surface flows of complex fluids. News Inf Pub Soc Rheol 74(2):6-9

McKinley G (2005b) Visco-elasto-capillary thinning and breakup of complex fluids. Massachusetts Institute of Technology, Department of Mechanical Engineering, MIT. Hatsopoulos Microfluidics Laboratory, Cambridge

McKinley G, Sridhar T (2002) Filament-stretching rheometry of complex fluids. Annu Rev Fluid Mech 34:375-415

McKinley G, Tripathi A (2000) How to extract the newtonian viscosity from capillary breakup measurements in a filament rheometer. J Rheol 44:653-670

Michud A, Hummel M, Haward S, Sixta H (2015) Monitoring of cellulose depolymerization in 1-ethyl-3-methylimidazolium acetate by shear and elongational rheology. Carbohydr Polym 117:355-363

Miller E, Clasen C, Rothstein J (2009) The effect of step-stretch parameters on capillary breakup extensional rheology (CaBER) measurements. Rheol Acta 48(6):625-639

Niedzwiedz K, Arnolds O, Willenbacher N, Brummer R (2009) How to characterize yield stress fluids with capillary breakup extensional rheometry (CaBER). Appl Rheol 19(4):41969

Oliveira M, McKinley G (2005) Iterated stretching and multiple beads-on-string phenomena in dilute solutions of highly extensible flexible polymers. Phys Fluids 17(7):071704

Oliveira M, Yeh R, McKinley G (2006) Iterated stretching, extensional rheology and formation of beads-on-string structures in polymer solutions. J Non-Newton Fluid Mech 137(1-3):137-148

Papageorgiou D (1995) On the breakup of viscous liquid threads. Phys Fluids 7:1529

Patruyo L, Muller A, Saez A (2002) Shear and extensional rheology of solutions of modified hydroxyethyl celluloses and sodium dodecyl sulfate. Polymer 43:6481-6493

Plog J, Kulicke W, Clasen C (2005) Influence of the molar mass distribution on the elongational behaviour of polymer solutions in capillary breakup. Appl Rheol 15:28-37

Rodd L, Scott T, Cooper-White J, Mckinley G (2005) Capillary break-up rheometry of low-viscosity elastic fluids. Appl Rheol 15:12-27

Rodriguez-Rivero C, Hilliou L, Martin del Valle E, Galan M (2014) Rheological characterization of commercial highly 
viscous alginate solutions in shear and extensional flows. Rheol Acta 53:559-570

Sachsenheimer D, Hochstein B, Willenbacher N (2014) Experimental study on the capillary thinning of entangled polymer solutions. Rheol Acta 53(9):725-739

Sattler R, Wagner C, Eggers J (2008) Blistering pattern and formation of nanofibres in capillary thinning of polymer solutions. Phys Rev Lett 100:164502

Sattler R, Gier S, Eggers J, Wagner C (2012) The final stages of capillary break-up of polymer solutions. Phys Fluids 24:023101

Sharma V, Haward S, Serdy J, Keshavarz B, Soderlund A, Threlfall-Holmes P et al (2015) The rheology of aqueous solutions of ethyl hydroxy-ethyl cellulose (EHEC) and its hydrophobically modified analogue (hmEHEC): extensional flow response in capillary break-up, jetting (ROJER) and in a cross-slot extensional rheometer. Soft Matter 11:3251-3270

Smolka L, Belmonte A (2006) Charge screening effects on filament dynamics in xanthan gum solutions. J Non-Newton Fluid Mech 137(1-3):103-109

Stelter M, Brenn G, Yarin A, Singh R, Durst F (2000) Validation and application of a novel elongational device for polymer solutions. J Rheol 44:595-616

Stelter M, Brenn G, Yarin A, Singh R, Durst F (2002) Investigation of the elongational behavior of polymer solutions by means of an elongational rheometer. $\mathrm{J}$ Rheol 46(2):507-528

Storz H, Zimmermann U, Zimmermann H, Kulicke W (2010) Viscoelastic properties of ultra-high viscosity alginates. Rheol Acta 49(2):155-167

Szabo P, McKinley G, Clasen C (2012) Constant force extension of polymer solutions. J Non-Newian Fluid Mech 169-170:26-41

Tirtaatmadja V, McKinley G, Cooper-White J (2006) Drop formation and breakup of low viscosity elastic fluids: effects of molecular weight and concentration. Phys Fluids 18(4):043101

Tripathi A, Spiegelberg S, McKinley G (2000) Studying the extensional flow and breakup of complex fluids using filament rheometers. In: XIIIth international congress on rheology, vol 3. Cambridge, pp 55-57

Tripathi A, Tam K, McKinley G (2006) Rheology and dynamics of associative polymers in shear and extenion: theory and experiments. Macromolecules 39(5):1981-1999

Yarin A (1993) free liquid jets and films: hydrodynamics and rheology, 1st edn. Longman, Harlow and Wiley, New York

Zugenmaier P (2008) Crystalline cellulose and cellulose derivatives-characterization and structures, 1st edn. Springer, Clausthal-Zellerfeld 